\title{
BLOCK-BASED PHYSICAL MODELING WITH APPLICATIONS IN MUSICAL ACOUSTICS
}

\author{
Rudolf Rabenstein， Stefan Petrausch \\ Chair of Multimedia Communications and Signal Processing \\ University Erlangen-Nuremberg, Cauerstr. 7, D-91058 Erlangen, Germany \\ e-mail: rabe@LNT.de
}

\begin{abstract}
Block-based physical modeling is a methodology for modeling physical systems with different subsystems. Each subsystem may be modeled according to a different paradigm. Connecting systems of diverse nature in the discrete-time domain requires a unified interconnection strategy. Such a strategy is provided by the well-known wave digital principle, which had been introduced initially for the design of digital filters. It serves as a starting point for the more general idea of blockbased physical modeling, where arbitrary discrete-time state space representations can communicate via wave variables. An example in musical acoustics shows the application of block-based modeling to multidimensional physical systems.
\end{abstract}

Keywords: physical modeling, wave digital filters, sound synthesis.

\section{Introduction}

Complex natural or technical systems consist of a variety of components which interact with one another according to the laws of physics. Modeling a component of a physical system means to identify the relevant quantities (force, deflection, pressure, velocity, voltage, current, etc.), to set up a mathematical description, and to find a suitable discretization for the computational realization. At the end of this process stands a discrete-time and possibly a discrete-space algorithm for real-time simulation of the corresponding system component. Encapsulating the algorithm into an appropriate input-output description leads to a component model which processes output-samples from input-samples, preferably in real time and with no latency. Such a component model is also called a block, because it acts as a building block for larger systems.

Creating accurate and meaningful block models requires considerable expertise in physics, engineering, numerical mathematics, and computer science. It is therefore desirable to provide tested and veryfied modeling blocks in software libraries. Users of these libraries can then concentrate on the creation of complex models from the basic building blocks.

Since the components of a physical system may be quite different in nature, also their bocks may be modeled according to different paradigms. Any connection of such modeling blocks may therefore not rely on the compati- bility of their inner variables but only on the input-output samples at each time instant. Creating a correct interaction between component models therefore means to provide a meaningful connection between the input and output ports of each block in the system.

Block-based modeling of physical systems thus consists of two separate tasks: component modeling and model interaction. It is an established method in the simulation of one-dimensional systems for which a variety of simulation languages and graphical simulation tools exist (e.g., Simulink). However, there are a number of special requirements like distributed parameter systems, interactive human control, real-time operation, and low latency, which are rarely provided by standard simulation tools.

This contribution adopts a general view on model interaction for satisfying these special requirements. To exemplify the general results, musical acoustics is chosen as an application scenario. The presentation is based on elements from the keynote talk (Rabenstein and Petrausch, 2006) and the magazine article (Rabenstein et al., 2007).

Section 2 presents some general concepts of blockbased physical modeling with special consideration of the wave digital principle. Section 3 introduces the idea of block-based physical modeling with state space and wave digital models. A detailed example in Section 4 shows the application of these ideas to a musical instrument. 


\section{Block-based physical modeling with wave-based interconnections}

2.1. Signals, ports and waves. When dealing with physical models and their realization with discrete-time systems, it is important to distinguish the domain of signals and systems from the domain of physics. Some concepts from these domains are presented here. In particular, signals, ports and wave variables are reviewed.

2.1.1. Signals. Signals are variable quantities which carry information. They have no immediate physical reality. However, for transmission, storage and processing, signals are usually represented by physical quantities like electrical voltage, magnetical flux, light intensity, etc. Systems for signal processing are often described by signal flow graphs. In a signal flow graph, blocks are fed by input signals and respond with the corresponding output signals. The output of one block serves as input for one or more other blocks. The assumption in a signal flow graph representation is that the output signal of one block does not change its values when it is connected to another block as an input signal.

Although blocks in a signal flow graph are described by mathematical operators (e.g., multipliers, integrators, input-putput-mappings, transfer functions, etc.), they may also be realized by physical systems, e.g., electrical circuits. However, care has to be taken not to violate the above assumption. Using block realizations with high input impedance and low output impedance ensures that their output voltages are valid representations of the corresponding signals.

2.1.2. Ports. Interconnections of physical models are described by ports. A port is a pair of variables which serve for communication with other blocks. The physical description of a system leads to potential and flow variables, also referred to as across and through variables. Typical port variables are voltage and current, pressure and flow, or force and velocity. In electrical circuits, voltage and current obey the Kirchhoff laws. Similar balance relations exist for mechanical, hydraulical, pneumatic, and other physical regimes. Therefore, these physical variables are also called Kirchhoff variables or simply Kvariables.

For continuous-time systems the balance equations are satisfied at any time instant. Since potential and flow variables at a port depend on each other, they cannot be grouped into input and output signals. Connecting two ports will in general change potential and flow variables at both ports.

2.1.3. Delay-free loops. For discrete-time approximations, the differential operators in a physical description are replaced by difference equations. Connecting two discrete-time systems may lead to delay free loops, which inhibit the implementation by digital computation.

Consider two blocks $f_{i}, i=1,2$ with input $v_{i}(k)$ and output $y_{i}(k)$, where $k$ is the discrete time variable. If both blocks contain a direct path between the input and the output, then the sequence

$$
\begin{aligned}
& y_{1}(k)=f_{1}\left\{v_{1}(k)\right\}, \\
& v_{2}(k)=y_{1}(k), \\
& y_{2}(k)=f_{2}\left\{v_{2}(k)\right\}, \\
& v_{1}(k)=y_{2}(k)
\end{aligned}
$$

is not computable. Although each discrete-time model $f_{i}$ may be correctly implemented, the blocks cannot be directly connected. A naive way to overcome this problem is to insert a delay in the interconnection, e.g., by computing $y_{1}(k)=f\left\{v_{1}(k-1)\right\}$, but this crude measure changes the dynamics of the systems in an unpredictable way.

2.1.4. Wave variables. A more elegant and mathematically rigorous way to avoid delay-free loops involves wave variables. They are defined as a linear combination of potential and flow variables, e.g., voltage $u(t)$ and current $i(t)$ in a electrical circuit. The compatibility of the physical units is ensured by the port resistance $R_{\mathrm{p}}$ as

$$
\begin{aligned}
& a(t)=u(t)+R_{\mathrm{p}} i(t), \\
& b(t)=u(t)-R_{\mathrm{p}} i(t) .
\end{aligned}
$$

In accordance with physical intuition, $a(t)$ is called the incident wave and $b(t)$ the reflected wave. They are also called wave variables or $\mathrm{W}$-variables. Figure 1 shows the $\mathrm{K}$-variables $u(t)$ and $i(t)$ as well as the wave variables $a(t)$ and $b(t)$ at a one-port with port resistance $R_{\mathrm{p}}$.

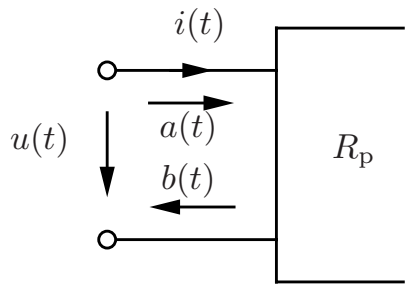

Fig. 1. K- and $\mathrm{W}$-variables at a one-port with the port resistance $R_{\mathrm{p}}$.

2.1.5. Interconnection of ports with wave variables. To interconnect ports with wave variables, relations have to be established between the voltages and currents and the corresponding waves. This procedure is shown in Fig. 2 for the simplest case, the interconnection of two one-ports with the port resistances $R_{1}$ and $R_{2}$. The Kvariables are restricted by the Kirchhoff laws as

$$
u_{1}(t)=u_{2}(t), \quad i_{1}(t)=-i_{2}(t) .
$$




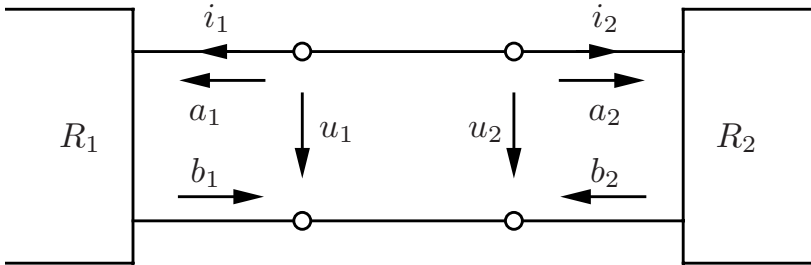

Fig. 2. Interconnection of two one-ports.

Also the wave variables have to satisfy certain relations. The definitions of the wave variables for Fig. 2] and their sum and difference are compiled below for $n=1,2$ and $G_{n}=R_{n}^{-1}$ (the time index $t$ is omitted):

$$
\begin{aligned}
a_{n} & =u_{n}+R_{n} i_{n}, \quad 2 u_{n}=a_{n}+b_{n}, \\
b_{n} & =u_{n}-R_{n} i_{n}, \quad 2 i_{n}=G_{n}\left(a_{n}-b_{n}\right) .
\end{aligned}
$$

Expressing the Kirchhoff laws (3) for $u_{n}$ and $i_{n}$ in terms of the wave variables $a_{n}$ and $b_{n}$ results in the matrix equation

$$
\begin{array}{r}
{\left[\begin{array}{cc}
1 & -1 \\
G_{1} & G_{2}
\end{array}\right]\left[\begin{array}{l}
a_{1} \\
a_{2}
\end{array}\right]-\left[\begin{array}{cc}
-1 & 1 \\
G_{1} & G_{2}
\end{array}\right]} \\
=\left[\begin{array}{l}
b_{1} \\
b_{2}
\end{array}\right] \\
=\left[\begin{array}{l}
0 \\
0
\end{array}\right] .
\end{array}
$$

It can be solved for the incident waves $a_{n}$ in terms of the reflected waves $b_{n}$ as

$$
\begin{gathered}
{\left[\begin{array}{l}
a_{1} \\
a_{2}
\end{array}\right]=\left(\left[\begin{array}{ll}
\alpha_{1} & \alpha_{2} \\
\alpha_{1} & \alpha_{2}
\end{array}\right]-\mathbf{I}_{2}\right)\left[\begin{array}{l}
b_{1} \\
b_{2}
\end{array}\right],} \\
\alpha_{n}=\frac{2 G_{n}}{G_{1}+G_{2}},
\end{gathered}
$$

where $\mathbf{I}_{2}$ is the $2 \times 2$ identity matrix. Such relations between incident and reflected waves are also called scattering relations.

When the ports in Fig. 2are not expressed in terms of the port variables but in terms of the wave variables (wave ports), then the validity of (6) has to be ensured by a special block. It can be regarded as an impedance matcher between the port resistances and is called the adaptor. Figure 3 shows the two ports from Fig. 2 as wave ports with the corresponding two-port adaptor. Note that the connections in Fig. 3 are expressed in W-variables in contrast to the K-variables in Fig. 2 For the adaptor, the roles of the incident and reflected waves are interchanged such that its defining equations are (see (6)

$$
\begin{aligned}
& b_{1}^{\prime}=\left(\alpha_{1}-1\right) a_{1}^{\prime}+\alpha_{2} a_{2}^{\prime}, \\
& b_{2}^{\prime}=\alpha_{1} a_{1}^{\prime}+\left(\alpha_{2}-1\right) a_{2}^{\prime} .
\end{aligned}
$$

The interconnection of blocks in terms of Wvariables is an important component of the so-called wave digital principle described below.

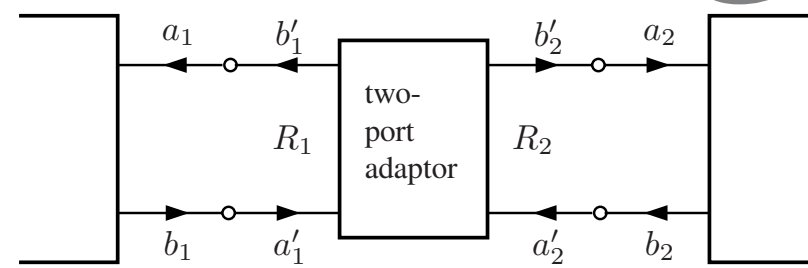

Fig. 3. Interconnection of two wave ports with a parallel adaptor.

2.2. Wave digital principle. The wave digital principle evolved as a method for designing digital filters (wave digital filters) from analog counterparts, which were described by ordinary differential equations (Fettweis, 1971). A unifying treatment of the theory and application of wave digital filters is given in the classical paper (Fettweis, 1986). Later this principle was extended to distributed parameter systems, described by partial differential equations (Fettweis and Nitsche, 1991). Modern descriptions of the wave digital principle as a tool for numerical integration and modeling are given in (Ochs, 2001; Bilbao, 2004; Välimäki et al., 2006; Smith, 2007). The following subsections present a short introduction to the basic idea of wave digital filters.

2.2.1. Simple example. A quick overview of the design of wave digital filters is given here by a very simple example: the charging of a capacitor by a voltage source with an internal resistance. The network description in the form of an RC-circuit is shown in Fig. 4. The timevarying voltage $u_{0}(t)$ of the source is the input and the voltage $u(t)$ across the capacitor is the output.

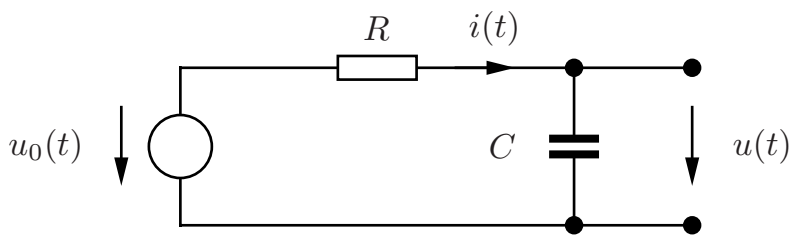

Fig. 4. Network description of an RC-circuit.

2.2.2. Energy storage element. The energy storage element in the RC-circuit is the capacitor which relates $u$ and $i$ by integration,

$$
u(t)=\frac{1}{C} \int_{-\infty}^{t} i(\tau) \mathrm{d} \tau .
$$

Numerical integration by the trapezoidal rule with the time step size $T$ performs the time discretization according to the bilinear transformation ( $k$ is the discrete time index) 


$$
\left.u((k+1) T)=u(k T)+\frac{T}{2 C}[i(k+1) T)+i(k T)\right] .
$$

However, this difference equation in terms of $\mathrm{K}$-variables is not computable, since neither $u((k+1) T)$ nor $i((k+$ 1)T $)$ is known at the time instant $(k+1) T$.

To prepare the transition to wave variables, the unknown quantities are sorted according to time,

$$
u((k+1) T)-\frac{T}{2 C} i((k+1) T)=u(k T)+\frac{T}{2 C} i(k T) .
$$

Then the discrete wave quantities are defined as

$$
\begin{aligned}
a[k] & =u(k T)+R_{\mathrm{p}} i(k T), \\
b[k] & =u(k T)-R_{\mathrm{p}} i(k T) .
\end{aligned}
$$

Their introduction is the key element of the wave digital principle. While the across and through variables $u$ and $i$ mutually depend on each other, there is a causal relation between the wave variables $a$ and $b$, which ensures the computability of the resulting structure. Expressing (12) in terms of the wave quantities and choosing the reference resistance as $R_{\mathrm{p}}=T / 2 C$ results in the simple relation (cf. also Table 1 )

$$
b[k]=a[k-1] .
$$

Thus the numerical integration (12) becomes computable by expressing the reflected wave $b[k]$ at the time instant $k$ by the incoming wave $a[k-1]$ at the previous time $k-1$.

Table 1 lists some selected elements of electrical networks and their wave digital counterparts. Since electrical networks are frequently used as an analogon for other networks (mechanical, thermal, flow), the more general term "Kirchhoff network" has been chosen.

The behavior of the wave digital equivalents follows from similar derivations as for the capacitor in (10) - 15). For all linear elements, the reflected wave $b[k]$ is given by known quantities or by the incident wave $a[k-1]$ from the previous time step. For non-linear elements, the relation between $a$ and $b$ depends on the type of non-linearity.

2.2.3. Wave digital filter. Now all required elements have been presented for the derivation of the wave digital filter equivalent of the RC-circuit from Fig. 4. It is shown again in Fig. 5 with the decomposition into its two basic components: a voltage source with internal resistance and a capacitor. In addition, the incident and reflected waves of both components are shown.

The port resistances are $R$ and $T / 2 C$. They will be different in general, and thus a two-port adaptor according to Fig. 2 is required. Figure 6 shows the resulting wave digital filter structure. Obviously, the reflected waves of the two one-port elements are the incident waves of the adaptor (indicated by primes) and vice versa.

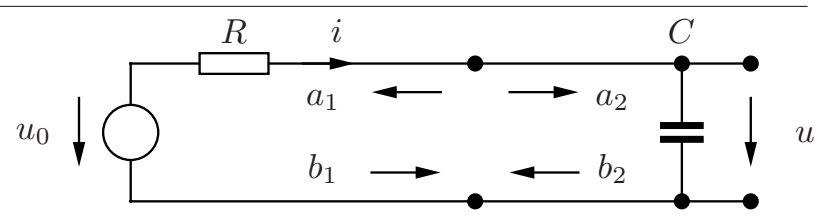

Fig. 5. RC-circuit with decomposition into basic components and wave variables.

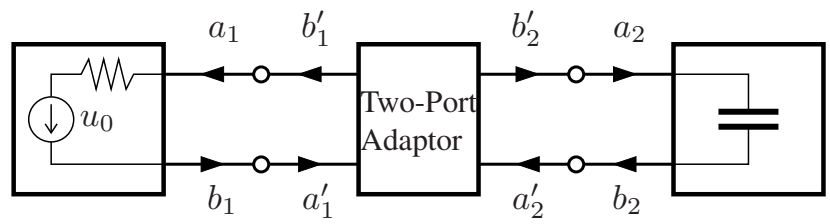

Fig. 6. Wave digital filter structure of the RC-circuit from Fig. 5

Finally, the computational structure of the resulting discrete-time system is shown in Fig. 7. It consists of adders, multipliers and a delay only. The two-port adaptor is represented by the equations from Fig. 2 with $a_{1}^{\prime}=u_{0}$. The reflected wave $b_{1}^{\prime}$ is not required since the voltage source needs no incident wave (see Table 1). The delay between the incident and the reflected wave at the capacitor according to (15) is indicated by $z^{-1}$, the $z$-transform equivalent of a delay. The output voltage $u$ follows from

$u_{0}$

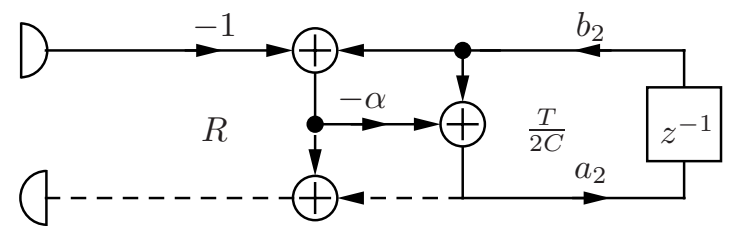

Fig. 7. Computational structure of the resulting discrete-time system.

the definition of the wave variables $13[14$ as

$$
u=\frac{1}{2}\left(a_{2}+b_{2}\right)
$$

Now it is straightforward to derive the transfer function of the discrete-time implementation as the relation between the $z$-transforms $U(z)$ and $U_{0}(z)$ of the output and the input signal, respectively,

$$
H(z)=\frac{U(z)}{U_{0}(z)}=\frac{\alpha}{2} \frac{z+1}{z-(1-\alpha)} .
$$

This transfer function can also be obtained from the continuous-time transfer function of the RC-circuit by a bilinear transformation. However, the wave digital principle provides not only the transfer function of the RCcircuit's discrete-time counterpart but also its internal structure. The algorithmic advantages of this strategy are discussed in detail in (Fettweis, 1986).

To apply the presented method also to larger networks, these have to be decomposed into serial and parallel arrangements of their network elements. Instead of 


\begin{tabular}{l|l||l|l|l|l}
\multicolumn{2}{c||}{ Kirchhoff network element } & \multicolumn{3}{c}{ Wave digital filter equivalent } \\
\hline Network Element & Behavior & Symbol & Behavior \\
\hline \hline
\end{tabular}

Table 1. Kirchhoff network elements and their wave digital counterparts. From top to bottom: resistor, inductance, capacitance, voltage source with internal resistance, non-linear element.

the two-port adaptor, general multi-port adaptors are required (Fränken et al., 2005; Sarti and Sanctis, 2006).

\subsubsection{Summary of the one-dimensional wave digi-} tal approach. The basic elements of the wave digital approach have been presented using a most simple example. For an in-depth coverage of the fundamental theory and implementation of wave digital filters, see, e.g., (Fettweis, 1986). The most prominent features are now briefly summarized:

- The wave digital approach is a method for the discretization of continuous-variable systems given by a physical description of their internal structure, e.g., an electrical network.

- It preserves not only the stability of the input-output system, but also the internal physical structure and its properties.

- If the continuous-variable system is passive, then also the corresponding discrete wave digital filter structure has the same property. This is also true in the presence of certain finite wordlength effects of a computer implementation.

Due to these properties, the wave digital approach is a powerful candidate for the modeling of continuous-time systems beyond lumped-parameter networks. Applications to distributed parameter systems were discussed, e.g., in (Fettweis and Nitsche, 1991; Fettweis, 1992; Rabenstein and Trautmann, 2003)

The theory of the wave digital principle is well founded on the theory of analog electrical networks, signals and systems, and digital signal processing. After many refinements and extensions (even to the theory of relativity (Fettweis, 2002)), it can be considered a rather mature field. Recently, also design methodologies and computer programs for the automated generation of wave digital structures were developed (Sanctis, Sarti and Tubaro, 2003; Sanctis, Sarti, Scarparo and Tubaro, 2005; Vollmer, 2005; Fränken et al., 2005; Sarti and Sanctis, 2006). However, in its present form the wave digital principle is an all-or-nothing strategy, since the above design methods require to design the complete system from scratch. Although the wave digital principle allows great flexibility 
within its framework, there are no provisions to consider models or simulation methods from outside the wave digital world. The next section presents an approach in this direction.

\section{Block-based physical modeling}

3.1. Beyond the wave digital principle. The wave digital principle as described above offers many advantages but it also puts severe restrictions on the designer. As a block-based modeling approach, it permits only blocks that have been designed according to this principle. There is no freedom to include subsystems adhering to a different modeling paradigm or to reuse an existing simulation code. It would be attractive to alleviate this restriction by permitting also other discretization strategies according to other modeling paradigms. Of course, the preservation of passivity and stability is then no more guaranteed by the wave digital principle. It has to be ensured for each block individually.

To make an arbitrary model suitable for interconnection with wave variables, it has to be augmented with an interface that converts its inputs and outputs to wave variables and vice versa. Such an interface is called a $K$ - $W$ converter (Petrausch and Rabenstein, 2005b). Similar to an adaptor which connects wave digital blocks with different port resistances, a K-W-converter connects an arbitrary model to an adaptor. Mixed modeling with digital waveguides and finite difference structures was also discussed in (Karjalainen and Erkut, 2004; Rabenstein et al., 2007).

This extension of the wave digital principle is called block-based physical modeling. Block-based means that different subsystems are modeled as individual blocks. Physical modeling means that the discrete-time and possibly discrete-space modeling blocks are derived from a physical description in terms of ordinary or partial differential equations. In this sense, the wave digital principle is a block-based physical modeling method, although a very special one. In general, block-based physical modeling permits also non-wave digital blocks or even only non-wave digital blocks if they are connected via $\mathrm{K}-\mathrm{W}$ converters and wave variables.

3.2. Wave ports. It remains to specify the idea of a $\mathrm{K}$-W-converter. Here it is shown how a very wide class of models can be equipped with a wave port, i.e., a connection to the wave digital world. No special modeling paradigm is assumed. All discretizations which represent a continuous lumped or distributed parameter system by a discrete-time state space representation are permitted.

Consider a state space representation of a discretetime system with the vector of inputs $\mathbf{v}[k]$, the vector of outputs $\mathbf{y}[k]$ and the state vector $\mathbf{z}[k]$. The discrete time index is $k$. We have

$$
\begin{aligned}
\mathbf{z}[k+1] & =\mathbf{A} \mathbf{z}[k]+\mathbf{B} \mathbf{v}[k], \\
\mathbf{y}[k] & =\mathbf{C} \mathbf{z}[k]+\mathbf{D} \mathbf{v}[k] .
\end{aligned}
$$

Now define the vectors of incident and reflected waves $\mathbf{a}[k]$ and $\mathbf{b}[k]$ with the matrix of port resistances $\mathbf{R}$ and the identity matrix $\mathbf{I}$ :

$$
\left[\begin{array}{l}
\mathbf{a}[k] \\
\mathbf{b}[k]
\end{array}\right]=\left[\begin{array}{rr}
\mathbf{I} & \mathbf{R} \\
\mathbf{I} & -\mathbf{R}
\end{array}\right]\left[\begin{array}{l}
\mathbf{y}[k] \\
\mathbf{v}[k]
\end{array}\right] .
$$

This definition can be solved for the quantities which should leave the wave port, i.e., for the input $\mathbf{v}[k]$ of the state space structure and for the reflected wave $\mathbf{b}[k]$ into the wave digital world. The result defines a first version of the $\mathrm{K}$-W-converter called the $\mathrm{K}-\mathrm{W}$-converter $\mathrm{I}$. We have

$$
\left[\begin{array}{l}
\mathbf{v}[k] \\
\mathbf{b}[k]
\end{array}\right]=\left[\begin{array}{cc}
-\mathbf{R}^{-1} & \mathbf{R}^{-1} \\
2 \mathbf{I} & -\mathbf{I}
\end{array}\right]\left[\begin{array}{l}
\mathbf{y}[k] \\
\mathbf{a}[k]
\end{array}\right] .
$$

However, comparing (19) and the top equation from 21 shows that the combination of the state space structure and $\mathrm{K}-\mathrm{W}$-converter I creates a delay-free loop for $\mathbf{D} \neq \mathbf{0}$. Therefore the $\mathrm{K}-\mathrm{W}$-converter I is suitable only for discrete systems with $\mathbf{D}=\mathbf{0}$ (see Fig. 8). Since it contains a direct path between incident and reflected waves, it must be connected to an adaptor with a reflection-free port.

To derive a version of the $\mathrm{K}-\mathrm{W}$-converter which is suitable for $\mathbf{D} \neq \mathbf{0}$, the output equation of the state space representation is inserted into the definition of the wave variables,

$$
\begin{aligned}
\mathbf{a}[k] & =\mathbf{C z}[k]+(\mathbf{D}+\mathbf{R}) \mathbf{v}[k], \\
\mathbf{b}[k] & =\mathbf{C z}[k]+(\mathbf{D}-\mathbf{R}) \mathbf{v}[k] .
\end{aligned}
$$

Choosing the port resistance $\mathbf{R}=\mathbf{D}$ yields a reflected wave $\mathbf{b}[k]$ in (23) without any instantaneous feedback. As the state vector $\mathbf{z}[k]$ only depends on past values (see (18)), a delayed reflected wave $\mathbf{b}[k]$ results. Thus the set of equations

$$
\begin{aligned}
\mathbf{z}[k+1] & =\mathbf{A} \mathbf{z}[k]+\mathbf{B} \mathbf{v}[k], \\
\mathbf{b}[k] & =\mathbf{C z}[k]
\end{aligned}
$$

constitutes a state space representation with a built-in Wvariable output for $\mathbf{b}[k]$, also called the wave port. Setting $\mathbf{R}=\mathbf{D}$ in (22) and (23), and solving for $\mathbf{v}[k]$ and $\mathbf{b}[k]$ gives

$$
\left[\begin{array}{c}
\mathbf{v}[k] \\
\mathbf{b}[k]
\end{array}\right]=\left[\begin{array}{cc}
-\frac{1}{2} \mathbf{D}^{-1} & \frac{1}{2} \mathbf{D}^{-1} \\
\mathbf{I} & 0
\end{array}\right]\left[\begin{array}{c}
\mathbf{C z}[k] \\
\mathbf{a}[k]
\end{array}\right],
$$

which constitutes the K-W-converter of type II (see Fig. 9. 


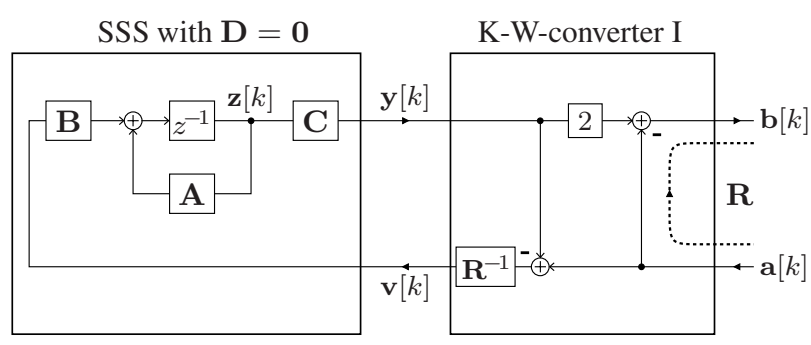

Fig. 8. State space structure (SSS) without direct feedback $(\mathbf{D}=\mathbf{0})$ and a K-W-converter of type I with an arbitrary port resistance $\mathbf{R}$. The instantaneous feedback from the incident to the reflected wave is accentuated by the dashed line.

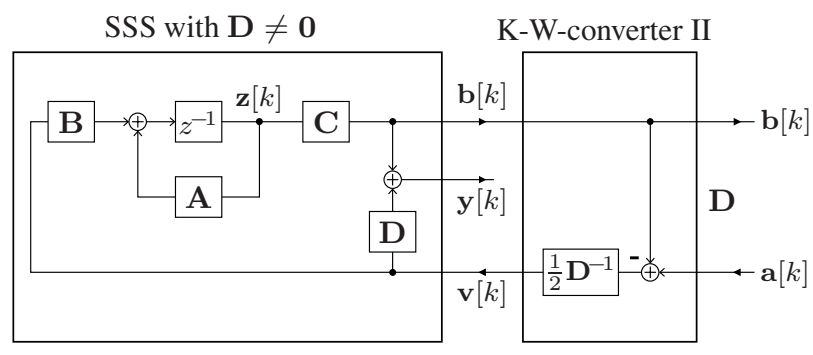

Fig. 9. Connection of a general linear K-variable model in the state space structure to $\mathrm{W}$-variables.

The introduction of the $\mathrm{K}-\mathrm{W}$-converters of types I and II is the key to the connection of models in a state space structure to wave variables. Using the wave digital interconnection strategy, an arbitrary number of such models may be connected with each other, with wave digital equivalents of network elements, and with other models using wave ports. Also existing models can be reused when an appropriate $\mathrm{K}-\mathrm{W}$-converter is attached. Compared with the wave digital principle introduced before, the range of possible blocks for model building is greatly enhanced. An example is presented in the next section.

\section{Example}

The construction of complex systems with different kinds of models is now discussed in greater detail. To this end, the block-based physical modeling approach presented above is applied to a problem from the area of musical instrument modeling.

4.1. Musical instrument modeling. The modeling and simulation of musical instruments for digital sound synthesis plays an increasing role in computer music and multimedia applications. This field is chosen as an example because it comprises a number of tough problems which are also encountered in other areas of simulation and modeling:
- Musical instruments are composed of different subsystems which are described by linear and non-linear ordinary and partial differential equations with timevarying parameters.

- Digital sound synthesis requires real-time computation of the above models under consumer oriented hardware and cost constraints.

- A digital musical instrument is a typical human-inthe-loop system which requires low latency. Typical values are some ten milliseconds from sensor input (e.g., a keyboard) to sound output.

4.2. Model of a brass instrument. The ability to model complex structures is now highlighted by a model of a brass instrument. Figure 10 shows the profile of a brass instrument with its four essential parts. The player's lips are the excitation mechanism and require a non-linear model. The mouthpiece is a resonator with a bandpass characteristic. The cylindrical air-column acts as an acoustic waveguide, and the horn matches the acoustic impedance to the free field. Both the air-column and the horn are distributed parameter systems and require partial differential equations as mathematical models. However, for an efficient realization, the differences in mathematical complexity between the air-column model and the horn model should be exploited. These models are discussed below based on (Krach et al., 2004; Petrausch and Rabenstein, 2005a).

4.2.1. Lip model. When playing a brass instrument, the player interacts with the instrument by using his lips, which are pressed against the mouthpiece. Thereby the lips behave as an oscillator that excites the air column inside the instrument. The lip oscillation is supported with energy provided by the player himself and reflections coming back from the instrument. The lip model is chosen to provide the behavior of lips towards a brass instrument in general and neglects details.

Physical models of the player's lips are usually formulated as pressure controlled valves, see, e.g., (Fletcher, 1993; Vergez and Rodet, 1997). Here a basic upward striking model is used as shown in Fig. 11 A mathematical description of the model is given by the following ODE:

$$
m_{0} \ddot{x}_{1}(t)+r_{0} \dot{x}_{1}(t)+k_{0} x_{1}(t)=\gamma\left(P_{\mathrm{s}}-p_{1}(t)\right),
$$

where $x_{1}$ is the opening of the lip, $m_{0}$ denotes the mass of the lip, $r_{0}$ is a damping constant and $k_{0}$ stands for the spring constant. Here $\gamma$ is a constant related to the geometric details of the lip model, $P_{\mathrm{s}}$ indicates the blowing pressure inside the mouth and $p_{1}$ is the pressure inside the mouthpiece. The volume flow $u_{1}$ entering the mouthpiece is set to zero except when the condition $\left(x_{1}(t)>\right.$ 


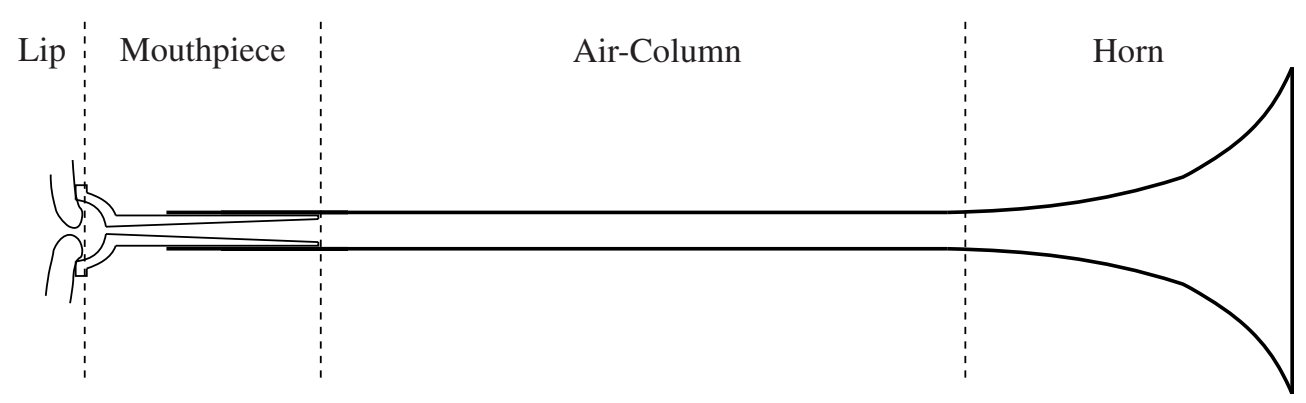

Fig. 10. Profile of a brass instrument. Details of the shape vary with the instrument. The model consists of four essential parts: the player's lips, the mouthpiece, the cylindrical air-column, and the horn.

$0) \wedge\left(P_{\mathrm{S}}-p_{1}(t)>0\right)$ is fulfiled. Then it is computed with

$$
u(t)=l \sqrt{\frac{2}{\varrho_{0}}} x_{1}(t) \sqrt{P_{\mathrm{s}}-p_{1}(t)} .
$$

The parameter $l$ is a constant describing the width of the lip and $\varrho_{0}$ is the static density of air.

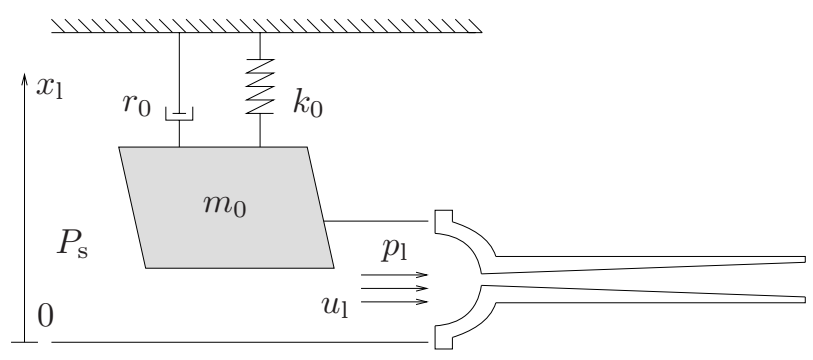

Fig. 11. Physical model for the lips of a brass player.

4.2.2. Mouthpiece model. The mouthpiece is the connection between the lips of the player and the air column. There are brass mouthpieces in various shapes and sizes. The details vary with the style of the instrument and the player's preferences, but all mouthpieces have the common general design shown in Fig. 12, The player presses the lips against the surface of the mouthpiece cup, which has a characteristic volume $V_{\mathrm{b}}$. A narrower passage of the diameter $S_{\mathrm{c}}$ and the length of $l_{\mathrm{c}}$ connects the cup to the main bore of the instrument.

A simple physical model of a mouthpiece can be found in (Fletcher and Rossing, 1998). The analogous acoustic network depicted in Fig. 13 is used to describe the basic physical behavior of a brass mouthpiece. The cup is

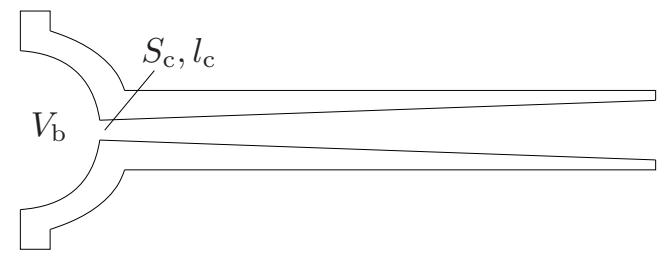

Fig. 12. Profile of a brass instrument mouthpiece.

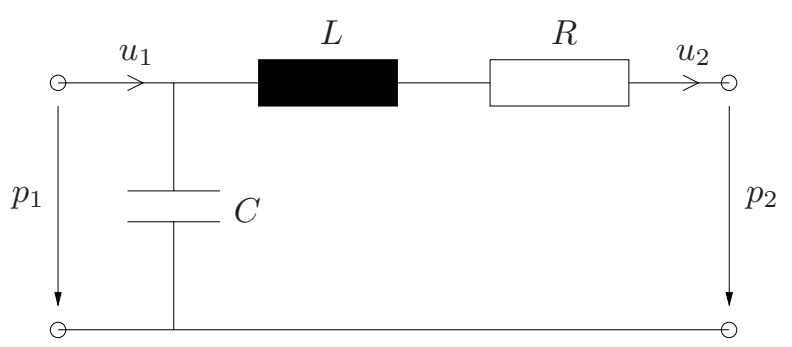

Fig. 13. Analogous acoustic network for a brass mouthpiece.

modeled as an acoustic compliance $C$ and the passage of constriction as an acoustic inertance $L$. Lossy effects in the mouthpiece are included in the model with a dissipative element $R$. The impedance quantities denote the ratio of the pressure to the volume flow. The values $L$ and $C$ of the physical model can be computed from the mouthpiece geometry as

$$
C=\frac{V_{\mathrm{b}}}{\varrho_{0} c^{2}}, \quad L=\frac{\varrho_{0} l_{\mathrm{c}}}{S_{\mathrm{c}}},
$$

where $c$ is the speed of sound. Pressure $p_{1}$ and $u_{1}$ on the left-hand side correspond to $p_{1}$ and $u_{1}$ from the lip model.

The network from Fig. 13 is realized here as a classical wave digital filter as shown in Fig. 16 It uses the wave digital filter equivalents of the Kirchhoff network elements according to Table 1 along with three-port serial and parallel adaptors (see, e.g., (Fettweis, 1986) for details).

4.2.3. Air-column. The model for the cylindrical aircolumn is the acoustic wave equation in one spatial dimension. Its solution can be formulated in terms of forward and backward travelling waves (d'Alembert solution). A very simple and efficient realization of this model is a dual delay line or a digital wave guide (Smith, 1998). The delay lines consist of $M$ delay elements for the sampling instant $T$. Their number $M$ is given by the length $l_{\mathrm{a}}$ of the air column as $M=l_{\mathrm{a}} /(c T)$. For non-integer delays, the so-called fractional delay elements are required. 
4.2.4. Horn. The standard model for sound propagation in horns and flares is Webster's well-known horn equation, here formulated in terms of the particle velocity potential $\Phi(x, t)$,

$$
\Phi^{\prime \prime}(x, t)+\frac{A^{\prime}(x)}{A(x)} \Phi^{\prime}(x, t)=\frac{1}{c^{2}} \ddot{\Phi}(x, t) .
$$

Primes serve as a shorthand notation for spatial derivation while dots denote time derivatives. The particle velocity potential is related to the pressure $p_{\mathrm{h}}(x, t)$ and the particle velocity $v_{\mathrm{h}}(x, t)$ in the horn as given by $[33$.

Here, the shape of the brass instrument's horn is assumed to be close to a Bessel horn. The radius function $r$ of such a horn is given by

$$
r(x)=r_{\mathrm{h}} x^{-\varepsilon}, \quad \frac{A^{\prime}(x)}{A(x)}=-\frac{2 \varepsilon}{x},
$$

where $\varepsilon$ is the flare parameter of the horn and $r_{h}$ is a scaling factor. Then the model for the Bessel horn is

$$
\Phi^{\prime \prime}(x, t)-\frac{2 \varepsilon}{x} \Phi^{\prime}(x, t)-\frac{1}{c^{2}} \ddot{\Phi}(x, t)=0
$$

or, in terms of the port variables $\Phi^{\prime}(x, t)$ and $\dot{\Phi}(x, t)$,

$$
\mathbf{y}(x, t)=\left(\begin{array}{c}
\Phi^{\prime}(x, t) \\
\dot{\Phi}(x, t)
\end{array}\right)=\left(\begin{array}{c}
v_{\mathrm{h}}(x, t) \\
-\frac{1}{\rho_{0}} p_{\mathrm{h}}(x, t)
\end{array}\right)
$$

as

$$
\left[\mathbf{A}_{\mathrm{h}}+\mathbf{I} \frac{\partial}{\partial x}-\mathbf{C}_{\mathrm{h}} \frac{\partial}{\partial t}\right] \mathbf{y}(\mathbf{x}, t)=\mathbf{0}
$$

with the identity matrix I and

$$
\mathbf{A}_{\mathrm{h}}=\left(\begin{array}{cc}
-\frac{2 \varepsilon}{x} & 0 \\
0 & 0
\end{array}\right), \quad \mathbf{C}_{\mathrm{h}}=\left(\begin{array}{ll}
0 & c^{-2} \\
1 & 0
\end{array}\right) .
$$

A numerical method for models as in (34) is obtained by the functional transformation method. The procedure is based on an expansion of the operator $\mathbf{A}_{\mathrm{h}}+$ $\mathbf{I} \partial / \partial x$ in (34) into its eigenfunctions and is described in (Trautmann and Rabenstein, 2003; Krach et al., 2004). It results in an algorithmic structure according to Fig. 14 with the sampled physical quantities $\Phi^{\prime}[k]$ and $\dot{\Phi}[k]$ as input and output. The input weighting constants $b_{\mu}$, output weighting constants $c_{\mu}$ and eigenvalues $\beta_{\mu}$ result from (Krach et al., 2004; Petrausch and Rabenstein, 2004).

The physical port variables from Fig. 14 cannot be directly connected to the digital waveguide model for the air column. Therefore, the algorithmic structure is represented as a state space model according to Figs. 8 and 9 Here the matrix A turns out to be diagonal due to the parallel structure in Fig. 14

Connecting the physical variables to a K-Wconverter II as shown in Fig. 9 results in a horn implementation with a wave port as shown in Fig. 15. The coefficients in Figs. 14 and 15 are related by $\hat{b}_{\mu}=e^{-\beta_{\mu} T} b_{\mu}$ and $D=\sum_{\mu=1}^{N} b_{\mu} c_{\mu}$. In this form, the algorithmic model is suitable for connection to wave variables.

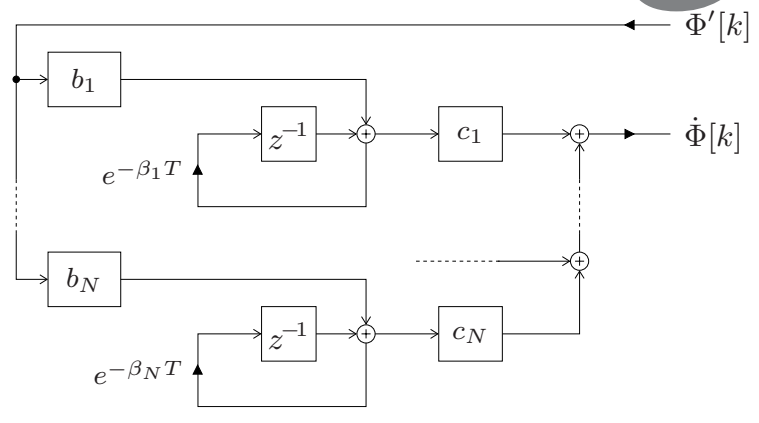

Fig. 14. Structure of the horn implementation with the physical variables $\Phi^{\prime}[k]$ and $\dot{\Phi}[k]$ as input and output.

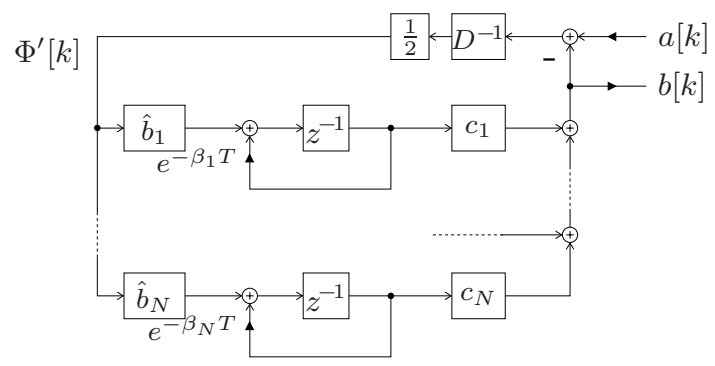

Fig. 15. Structure of the horn implementation with the wave variables $a[k]$ and $b[k]$ for input and output.

4.2.5. Block-based model. A block-based physical model is shown in Fig. 16. It is built from the algorithmic models discussed in Sections 4.2.1 to 4.2.4. The leftmost and the rightmost sections (lips and horn) are represented by K-variables, i.e., by potential and flow variables. The non-linear lip model according to (27) is formulated in terms of the pressure $p_{1}$ in the mouthpiece and the flow $u_{1}$ entering into the mouthpiece. The horn model is represented by the pressure $p_{\mathrm{h}}$ and the particle velocity $v$ according to 33 .

The two sections in the center (mouthpiece and aircolumn) are formulated in terms of wave variables (Wvariables). The linear lumped parameter model of the mouthpiece is realized by a standard wave digital filter structure shown in Fig. 16. The air-column is a distributed parameter system without loss and dispersion, and it is realized as a dual delay line, resembling the d'Alembert solution of the acoustic wave equation. The connection of both sections is easily accomplished by connecting the respective incident and reflected wave variables.

The connection of the lip model to the mouthpiece on the one hand and the connection of the air-column to the horn on the other hand require the transition between $\mathrm{K}$-variables to $\mathrm{W}$-variables and vice versa. The latter connection between the air-column and the horn is accomplished by attaching a wave port to the distributed parameter model of the horn as shown in Fig. 15 The connection between lip and mouthpiece models is based on a formulation of the non-linear model 27] in wave variables. De- 


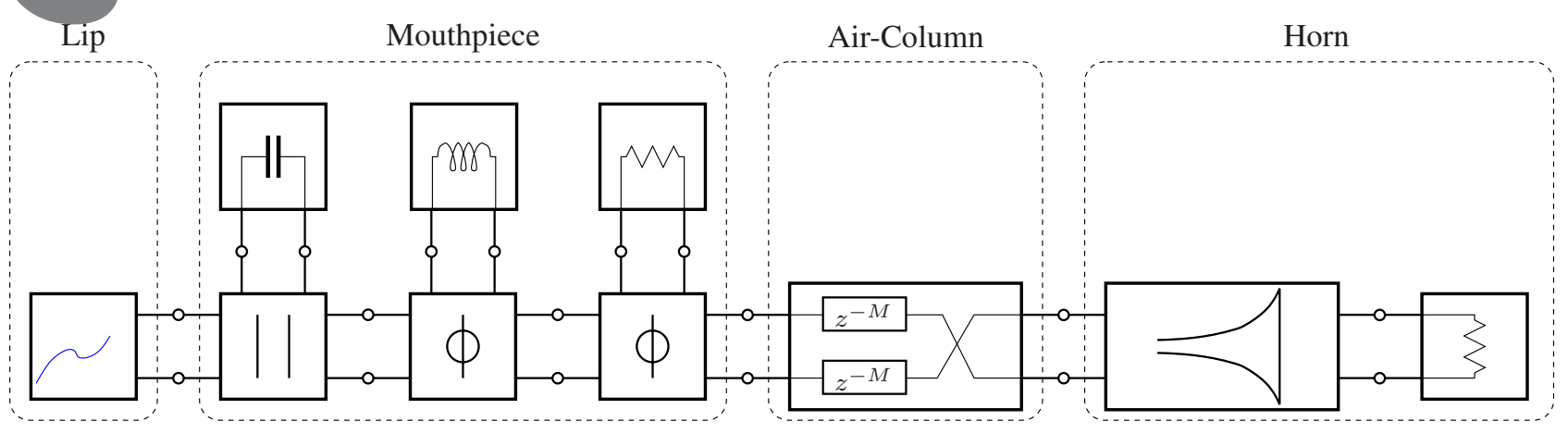

Fig. 16. Block-based model of a brass instrument. All blocks are compatible to the wave digital interaction topology. The horn is terminated by a simple resistor to simulate losses due to the sound radiation.

tails are not shown in Fig. 15 for simplicity but can be found in (Petrausch and Rabenstein, 2005a). The horn is terminated by a resistor to model the loss due to sound radiation. This resistor is considered as a boundary condition for (34) and determines the eigenfunctions $\beta_{\mu}$.

\section{Conclusion}

The distinction between input-output models and physical models is important for the construction of software libraries for modeling blocks. The connection of inputs and outputs does not change the values of the corresponding signals and thus does not require special consideration. The connection of physical models affects all port values and thus requires the consideration of the Kirchhoff laws for potential and flow variables. Another point to be regarded is the computability of a discrete-time model, i.e., avoiding delay-free loops. Both topics, the consideration of the Kirchhoff laws and avoiding delay-free loops, lead to a formulation of physical problems with wave variables. They form the basis of a general interconnection strategy for modeling blocks which encompasses models based on both Kirchhoff and wave variables.

A rather special example was considered as an application scenario, namely, a block-based model of a brass instrument. It was chosen because it combines linear and non-linear lumped parameter models along with linear distributed parameter models with different geometries. The presented block-based modeling method were applied to room acoustic simulation for the composition of complex geometries from basic building blocks.

\section{References}

Bilbao S. (2004). Wave and Scattering Methods for Numerical Simulation, Wiley \& Sons, Chichester.

Fettweis A. (1971). Digital filters related to classical filter networks, Archiv für Electronik und Übertragungstechnik (International Journal of Electronics and Communications) 25: 79-89.
Fettweis A. (1986). Wave digital filters: Theory and practice, Proceedings of the IEEE 74(2): 270-327.

Fettweis A. (1992). Multidimensional wave digital filters for discrete-time modelling of Maxwell's equations, International Journal of Numerical Modelling, Electronic Networks, Devices, and Fields 5: 183-201.

Fettweis A. (2002). The wave-digital method and some of its relativistic implications, IEEE Transactions on Circuits and Systems I 49(6): 862-868.

Fettweis A. and Nitsche G. (1991). Numerical integration of partial differential equations using principles of multidimensional wave digital filters, Journal of VLSI Signal Processing 3: 7-24.

Fletcher N. (1993). Autonomous vibration of simple pressurecontrolled valves in gas flows, Journal of the Acoustical Society of America 93(4): 2172-2179.

Fletcher N. H. and Rossing T. D. (1998). The Physics of Musical Instruments, 2nd Edn., Springer-Verlag, New York, NY.

Fränken D., Ochs J. and Ochs K. (2005). Generation of wave digital structures for networks containing multiport elements, IEEE Transactions on Circuits and Systems I, 52(3): 586596.

Karjalainen M. and Erkut C. (2004). Digital waveguides versus finite difference structures: Equivalence and mixed modeling, EURASIP Journal on Applied Signal Processing 2004(7): 978-989.

Krach B., Petrausch S. and Rabenstein R. (2004). Digital sound synthesis of brass instruments by physical modeling, Proceedings of the Conference on Digital Audio Effects (DAFx) 04, Naples, Italy, pp. 101-106.

Ochs K. (2001). Passive integration methods: Fundamental theory, International Journal of Electronic Communication (AEÜ) 55(3): 153-163.

Petrausch S. and Rabenstein R. (2004). A simplified design of multidimensional transfer function models, Proceedings of the International Workshop on Spectral Methods and Multirate Signal Processing (SMMSP2004), Vienna, Austria, pp. 35-40.

Petrausch S. and Rabenstein R. (2005a). Application of block based physical modeling for digital sound synthesis of 
brass instruments, Proceedings of the Conference on Forum Acusticum (FA 2005), Budapest, Hungary, pp. $703-$ 708 .

Petrausch S. and Rabenstein R. (2005b). Interconnection of state space structures and wave digital filters, IEEE Transactions on Circuits and Systems II 52(2): 90-93.

Rabenstein R. and Petrausch S. (2006). Block-based physical modeling, Proceedings of the 5th International Symposium on Mathematical Modelling (MATHMOD), Vienna, Austria, pp. 2-1-2-17.

Rabenstein R., Petrausch S., Sarti A., De Sanctis G., Erkhut C. and Karjalainen M. (2007). Blocked-based physical modeling for digital sound synthesis, Signal Processing Magazine 24(2): 42-54.

Rabenstein R. and Trautmann L. (2003). Towards a framework for continuous and discrete multidimensional systems, International Journal of Applied Mathematics and Computer Science 13(1): 73-85.

Sanctis G. D., Sarti A., Scarparo G. and Tubaro S. (2005). Automatic modeling and authoring of nonlinear interactions between acoustic objects, Proceedings of 4th International Workshop on Multidimensional Systems (NDS 2005), Wuppertal, Germany. Published on CD-ROM.

Sanctis G. D., Sarti A. and Tubaro S. (2003). Automatic synthesis strategies for object-based dynamical physical models in musical acoustics, Proceedings of the Conference on Digital Audio Effects (DAFx-03), London, UK, pp. DAFX-1DAFX-6.

Sarti A. and Sanctis G. D. (2006). Memory extraction from dynamic scattering junctions in wave digital structures, $\mathrm{Si}$ gnal Processing Letters 13(12): 729-732.
Smith J. O. (2007). Physical audio signal processing: For virtual musical instruments and digital audio effects, Technical report, Stanford University Center for Computer Research in Music and Acoustics, available at: http://ccrma.stanford.edu/ ${ }^{\sim}$ jos/pasp/pasp.html

Smith J. O. (1998). Principles of digital waveguide models of musical instruments, in M. Kahrs and K. Brandenburg, (Eds.), Applications of Digital Signal Processing to Audio and Acoustics, Kluwer Academic Publishers, Norwell, MA, USA, pp. 417-466.

Trautmann L. and Rabenstein R. (2003). Digital Sound Synthesis by Physical Modeling of Musical Instruments using Functional Transformation Models, Kluwer Academic/Plenum Publishers, Boston, MA.

Välimäki V., Pakarinen J., Erkut C. and Karjalainen M. (2006). Discrete time modeling of musical instruments, Reports on Progress in Physics 69(1): 1-78.

Vergez C. and Rodet X. (1997). Comparison of real trumpet playing, latex model of lips and computer model, Proceedings of the 1997 International Computer Music Conference, Thessaloniki, Greece. Published on CD-ROM.

Vollmer M. (2005). Automatic generation of wave digital structures for numerically integrating linear symmetric hyperbolic PDEs, Multidimensional Systems and Signal Processing 16(4): 369-396.

Received: 10 May 2006

Revised: 24 October 2006

Re-revised: 15 December 2006 
